\title{
Optimization of Experimental Conditions of Automated Glucuronidation Assays in Human Liver Microsomes Using a Cocktail Approach and Ultra-High Performance Liquid Chromatography-Tandem Mass Spectrometry
}

\author{
Justine Badée, Nahong Qiu, Neil Parrott, Abby C. Collier, Stephan Schmidt, and Stephen Fowler
}

Department of Pharmaceutics, Center for Pharmacometrics and Systems Pharmacology, University of Florida at Lake Nona, Orlando, Florida (J.B., S.S.); Pharmaceutical Sciences, Roche Pharma Research and Early Development, Roche Innovation Centre Basel, Basel, Switzerland (N.Q., N.P., S.F.); and Faculty of Pharmaceutical Sciences, University of British Columbia, Vancouver, British Columbia, Canada (A.C.C.)

Received September 6, 2018; accepted November 20, 2018

\section{ABSTRACT}

UDP-glucuronosyltransferase (UGT)-mediated metabolism is possibly the most important conjugation reaction for marketed drugs. However, there are currently no generally accepted standard incubation conditions for UGT microsomal assays, and substantial differences in experimental design and methodology between laboratories hinder cross-study comparison of in vitro activities. This study aimed to define optimal experimental conditions to determine glucuronidation activity of multiple UGT isoforms simultaneously using human liver microsomes. Hepatic glucuronidation activities of UGT1A1, UGT1A3, UGT1A4, UGT1A6, UGT1A9, UGT2B4, UGT2B7, UGT2B10, UGT2B15, and UGT2B17 were determined using cocktail incubations of 10 UGT probe substrates. Buffer components and cosubstrates were assessed over a range of concentrations including magnesium chloride $\left(\mathrm{MgCl}_{2} ; 0-10 \mathrm{mM}\right)$ and uridine 5' -diphosphoglucuronic acid (UDPGA; 1-25 mM) with either Tris- $\mathrm{HCl}$ or potassium phosphate buffer (100 mM, pH 7.4). Greater microsomal glucuronidation activity by different hepatic UGT isoforms was obtained using $10 \mathrm{mM} \mathrm{MgCl}$ and $5 \mathrm{mM}$ UDPGA with $100 \mathrm{mM}$ Tris$\mathrm{HCl}$ buffer. The influence of bovine serum albumin (BSA; $0.1 \%-2 \% \mathrm{w} / \mathrm{v}$ ) on glucuronidation activity was also assessed. Enzyme- and substratedependent effects of BSA were observed, resulting in decreased total activity of UGT1A1, UGT1A3, and UGT2B17 and increased total UGT1A9 and UGT2B7 activity. The inclusion of BSA did not significantly reduce the between-subject variability of UGT activity. Future in vitro UGT profiling studies under the proposed optimized experimental conditions would allow high-quality positive control data to be generated across laboratories, with effective control of a high degree of between-donor variability for UGT activity and for chemical optimization toward lower-clearance drug molecules in a pharmaceutical drug discovery setting.

\section{Introduction}

In recent years, the interest in glucuronidation reactions mediated via UDP-glucuronosyltransferase (UGT) enzymes has significantly increased as a clearance mechanism of new chemical entities (Di and Obach, 2015). In humans, UGTs comprise a large family of membrane-bound enzymes. UGTs play a major role in the detoxification and elimination of endogenous substrates (e.g., bilirubin, bile acid, and steroids) and xenobiotics (e.g., drugs, toxins, and carcinogens) by catalyzing the transfer of a glucuronic acid moiety from their cosubstrate, uridine 5 '-diphosphoglucuronic acid (UDPGA) (Tukey and Strassburg, 2000; Rowland et al., 2013).

In vitro glucuronidation studies are routinely conducted to determine the enzymes involved in conjugation as well as the fraction metabolized via this route using human liver microsomes (HLMs). Because of their reproducible nature, capacity for long-term storage, and rapid availability, HLMs are used as the first-line screening model for high-throughput

This work was supported by the Roche Postdoctoral Fellowship Program. https://doi.org/10.1124/dmd.118.084301.

S This article has supplemental material available at dmd.aspetjournals.org. assays for determination of UGT metabolism. They may be used to assess expression and activities within a population of individual donors (Miyagi and Collier, 2011; Gill et al., 2012; Walsky et al., 2012; Slovak et al., 2017). Methodological advances have in recent years improved the accuracy and reproducibility of microsomal UGT activity determination using selective or partially selective UGT probe substrates. This includes the optimization of incubation conditions for five important UGT isoforms (UGT1A1, UGT1A4, UGT1A6, UGT1A9, and UGT2B7) (Walsky et al., 2012). In addition, cocktail incubation approaches coupled with liquid chromatography-tandem mass spectrometry (MS/MS) quantification, typically under a low HLM concentration suited to inhibition assays, have been developed (Gagez et al., 2012; Joo et al., 2014; Seo et al., 2014; Gradinaru et al., 2015). The in vitro cocktail incubation of multiple substrates approach has been well described for determining the activity of cytochrome P450 enzymes in HLMs (Patten, 2006; Dixit et al., 2007; Pillai et al., 2013; Spaggiari et al., 2014). In contrast, determination of the glucuronidation activity of individual UGT isoforms can be challenging due to the lack of selective and validated UGT probe substrates (Donato et al., 2010). To date, a method for the simultaneous determination of glucuronidation activities

ABBREVIATIONS: BSA, bovine serum albumin; DMSO, dimethylsulfoxide; HLM, human liver microsome; MS/MS, tandem mass spectrometry; UDPGA, uridine 5'-diphosphoglucuronic acid; UGT, UDP-glucuronosyltransferase; UHPLC, ultra-high performance liquid chromatography. 
of major hepatic UGT isoforms (UGT1A1, UGT1A3, UGT1A4, UGT1A6, UGT1A9, UGT2B4, UGT2B7, UGT2B10, UGT2B15, and UGT2B17) has not been developed for use with microsomal preparations (Badée et al., 2018). This results in substantial interlaboratory technical and methodological differences that have hindered the crossstudy comparison of glucuronidation activity. Therefore, understanding of the differential contribution of analytical, technical, and betweensubject variability in glucuronidation activity remains challenging. The variations observed in experimental conditions can be listed as follows: 1) microsomal protein concentrations, 2) use of (validated and selective) UGT probe substrates, 3) incubation buffer to adjust and stabilize the desired $\mathrm{pH}$ of the reaction, 4) preincubation of microsomes with poreforming agents (e.g., alamethicin) or detergents, 5) a wide concentration range of UDPGA (1-30 mM) and differing buffer components such as magnesium chloride $\left(\mathrm{MgCl}_{2} ; 0-10 \mathrm{mM}\right)$ and 6) supplementation or not with bovine serum albumin (BSA; $0.1 \%-4 \%$ ).

Walsky et al. (2012) addressed the substrate characterization challenge and thereby proposed the best guide so far for in vitro glucuronidation assays to measure the glucuronidation activity of UGT1A1, UGT1A4, UGT1A6, UGT1A9, and UGT2B7 in HLMs. Glucuronidation activities were enhanced by conducting incubations of alamethicin-treated HLMs with Tris-HCl buffer (100 mM, pH 7.4) and $10 \mathrm{mM} \mathrm{MgCl}_{2}$. However, controversy exists on the use of BSA. The use of $2 \% \mathrm{BSA}$ was shown to enhance the glucuronidation activity of UGT1A9 and UGT2B7, which was explained by the sequestration of inhibitory long-chain fatty acids released during microsomal preparation and incubation. However, reduced or unchanged glucuronidation activity was observed for UGT1A1, UGT1A4, and UGT1A6 (Walsky et al., 2012). Therefore, the utility of BSA supplementation in the profiling of new drug candidate molecules needs to be addressed due to the competing effects of enzyme-selective enhancement versus compound-dependent reduction of free incubation concentration and reaction rates.

The objective of this study was to optimize the incubation conditions of in vitro UGT glucuronidation assays using hepatic microsomal preparations under conditions suited to UGT phenotyping for low- to moderate-clearance compounds. For this purpose, automated glucuronidation experiments were conducted to determine simultaneously the metabolic activity of major hepatic UGT isoforms by incubation of 10 UGT probe substrates in cocktails. First, we investigated the effect of a wide concentration range of cosubstrates and buffer components. Then we explored whether increasing BSA concentrations enhanced glucuronidation activity. Protein binding assays were carried out to correct activities for the unbound concentration of probe substrates. Finally, we evaluated whether the incubation of individual HLMs with BSA reduced between-subject variability.

\section{Materials and Methods}

Chemicals and Reagents. $\beta$-Estradiol, chenodeoxycholic acid, 5-hydroxytryptophol, propofol, gemfibrozil, zidovudine, amitriptyline, oxazepam (racemic), testosterone, dimethylsulfoxide (DMSO), alamethicin, UDPGA, and fatty acid free BSA $(\geq 98 \%)$ were purchased from Sigma-Aldrich (St. Louis, MO). Trifluoperazine was purchased from LKT laboratories (St. Paul, MN). $\beta$-Estradiol-3-glucuronide, chenodeoxycholic acid-glucuronide, trifluoperazineglucuronide, propofol-glucuronide, gemfibrozil-glucuronide, zidovudineglucuronide, and amitriptyline-glucuronide were purchased from Toronto Research Chemicals (Toronto, ON, Canada). 5-Hydroxytryptophol-glucuronide was purchased from Synlnnova (Edmonton, AB, Canada). Magnesium chloride was purchased from Merck (Darmstadt, Germany). Tris buffer was purchased from AppliChem GmbH (Darmstadt, Germany). Hexa-deuterated midazolam was obtained from Hoffmann-La Roche (Basel, Switzerland). High-purity acetonitrile for ultra-high performance liquid chromatography (UHPLC) coupled with MS/MS was obtained from BioSolve (Lexington, MA).
HLMs. Mixed-sex pooled HLMs derived from 150 donors (lot 38290, 75 male and 75 female donors) were purchased from Corning Life Sciences (Tewksbury, MA). Individual HLMs derived from single adult donors were purchased from Sekisui XenoTech (Kansas City, KS). The individual HLM donor panel comprised 15 donors; 14 donors were Caucasian and one was African American, six were male and nine were female, and the average age was 46.8 years (range, 21-74 years). Microsomes were stored at $-80^{\circ} \mathrm{C}$.

Automated Glucuronidation Assay Program. The highly flexible automated glucuronidation assay program used to perform these studies was developed at Hoffmann-La Roche Ltd. and implemented on a Tecan Fluent system (Tecan Group Ltd., Männedorf, Switzerland), which was controlled by Fluent Control software (version 1.5). The Tecan Fluent system was equipped with an eightchannel air displacement liquid handling arm using disposable tips for pipetting liquids, as well as a 96/384-multichannel arm using disposable tips and a robotic manipulator arm for transporting plates. Metabolism time course experiments were performed using the Tecan Fluent system, allowing the incubation of microsomal preparations with 11 probe substrates combined with up to 12 different enzyme/buffer/enzyme-specific cofactor conditions within one 96-well plate and up to eight incubation time points from 0.5 to 30 minutes.

Cocktail Incubations of UGT Probe Substrates. Glucuronide formation of $\beta$-estradiol, chenodeoxycholic acid, trifluoperazine, 5-hydroxytryptophol, propofol, gemfibrozil, zidovudine, amitriptyline, oxazepam, and testosterone was used to determine the hepatic metabolic activity of UGT1A1, UGT1A3, UGT1A4, UGT1A6, UGT1A9, UGT2B4/2B7, UGT2B7, UGT2B10, UGT2B15, and UGT2B17, respectively (Supplemental Fig. 1). Oxazepam was incubated as a racemic preparation of $S$ - and $R$-stereoisomers. $\beta$-Estradiol-3-glucuronide was used to assess the microsomal glucuronidation activity of UGT1A1.

Glucuronidation activities were simultaneously evaluated by incubating the UGT probe substrates in several cocktails, in a similar manner to other cocktail studies previously described (Gagez et al., 2012; Joo et al., 2014; Seo et al., 2014; Gradinaru et al., 2015). $\beta$-Estradiol was incubated alone due to inhibition and mass spectrometry interference effects that made it unsuitable for use in the cocktails. The glucuronidation rate of each substrate in the individual incubation was compared with that in the cocktail incubation to identify potential metabolic and analytical interferences between selected UGT probe substrates. The final substrate concentration was close to or below the corresponding Michaelis-Menten constant $\left(K_{\mathrm{m}}\right)$ reported in HLMs (Supplemental Table 1, references therein). The final concentration of the UGT substrates used was $5 \mu \mathrm{M}$ with the exception of zidovudine, for which $100 \mu \mathrm{M}$ was used. To minimize the effect of organic solvent on UGT activity in the incubation, the cocktails of substrates were dissolved in DMSO and serially diluted to the required concentrations. The final concentration of DMSO was $0.5 \%(\mathrm{v} / \mathrm{v})$.

Optimization of Incubation Buffer and $\mathbf{M g C l}_{\mathbf{2}}$ Concentration. Experimental settings for optimizing the incubation conditions of glucuronidation assays using 10 UGT probe substrates are summarized in Table 1. Pooled HLMs (at a fixed final microsomal protein concentration of $1 \mathrm{mg} / \mathrm{ml}$ ) were pretreated with alamethicin $(10 \mu \mathrm{g} / \mathrm{ml})$ for 30 minutes on ice at $4^{\circ} \mathrm{C}$. Quench solutions containing ice-cold acetonitrile with hexa-deuterated midazolam as the internal standard (final concentration of $500 \mathrm{ng} / \mathrm{ml}$ ) were placed in the individual reservoirs on the Tecan deck. One-hundred and sixty microliters of quench were transferred in

\section{TABLE 1}

Experimental settings for optimizing incubation conditions of an automated UGT phenotyping assay using a panel of 10 UGT probe substrates in HLMs

\begin{tabular}{ll}
\hline Experimental Condition & \multicolumn{1}{c}{ Value } \\
\hline Material & Pooled HLMs (150 donors) \\
& $15 \mathrm{HLMs}$ from single donors \\
Enzyme concentration & $1 \mathrm{mg} / \mathrm{ml}$ \\
Incubation volume & $0.4 \mathrm{ml}$ \\
Alamethicin treatment & $10 \mu \mathrm{g} / \mathrm{ml}$, for 30 min at $4^{\circ} \mathrm{C}$ \\
Preincubation step & $5 \mathrm{~min}$, shaking at $37^{\circ} \mathrm{C}$ \\
Incubation time & $0.5-30$ min, shaking at $37^{\circ} \mathrm{C}$ \\
Incubation buffer & Potassium phosphate $(100 \mathrm{mM}$ at pH 7.4) \\
& Tris- $\mathrm{HCl}(100 \mathrm{mM}$ at $\mathrm{pH} 7.4)$ \\
$\mathrm{MgCl}_{2}$ & $0,1,2,5,8$, and $10 \mathrm{mM}$ \\
$\mathrm{UDPGA}$ & $1,2.5,3.75,5,8,12,16$, and $25 \mathrm{mM}$ \\
$\mathrm{BSA}$ & $0 \%, 0.1 \%, 0.2 \%, 0.5 \%, 1.0 \%, 1.5 \%$, and $2 \% \mathrm{w} / \mathrm{v}$ \\
\hline
\end{tabular}


384-deep well plates. Two microliters of each test compound solution was added in the incubation mixtures and placed in a 96-well plate on the Tecan deck. Incubation mixtures containing alamethicin-treated HLMs, in either potassium phosphate or Tris-HCl buffer (100 mM, pH 7.4) and $\mathrm{MgCl}_{2}$ at different concentrations (0, 1, 2, 5, 8 , and $10 \mathrm{mM}$ ) were placed in a 96-well plate on the Tecan desk. Three-hundred and sixty microliters of the incubation solutions along with the 96-well plate containing UDPGA $(50 \mathrm{mM})$ were preincubated for 5 minutes with shaking $(800 \mathrm{rpm})$ at $37^{\circ} \mathrm{C}$.

After 5 minutes of preincubation, reactions were initiated by the addition of $40 \mu$ l UDPGA (final concentration of $5 \mathrm{mM}$ ) to obtain a final incubation volume of $0.4 \mathrm{ml}$. Reactions were terminated by transferring $80 \mu \mathrm{l}$ incubation mixture at each time point $(0.5,3.5,6.5,10,15,20,25$, and 30 minutes) into the 384-deep well plates preloaded with quench solutions. Plates were then sealed with adhesive aluminum sealing foils using an automated plate sealer (Agilent Technologies, Santa Clara, CA). Samples were centrifuged (Heraeus Multifuge $\times 3 \mathrm{R}$ centrifuge; Thermo Fisher Scientific, Waltham, MA) at $4^{\circ} \mathrm{C}$ and $6000 \mathrm{~g}$ for 10 minutes. Fifty microliters of supernatant was transferred into 384-well plates. All reaction rate calculations were performed using multiple timepoint data and following linear initial rate conditions. Glucuronidation reactions were carried out in triplicate, unless otherwise mentioned.

Optimal Concentration of UDPGA. UGT probe substrates were coincubated with UDPGA at different concentrations $(1,2.5,3.75,5,8,12,16$, and $25 \mathrm{mM})$ as well as with optimal incubation buffer $(100 \mathrm{mM}$ Tris- $\mathrm{HCl})$ and $10 \mathrm{mM} \mathrm{MgCl}$ concentration in pooled HLMs (at a fixed final microsomal protein concentration of $1 \mathrm{mg} / \mathrm{ml})$. All reactions were terminated after 3.5-15 minutes of incubation to ensure linearity and measurability using this microsomal protein concentration. Glucuronidation reactions were carried out in triplicate, unless otherwise mentioned.

Protein Binding to HLMs and BSA. Protein binding for all selected UGT probe substrates was measured at a low drug concentration and under optimized incubation conditions of the in vitro glucuronidation assays $\left(10 \mathrm{mMgCl}_{2}, 5 \mathrm{mM}\right.$ UDPGA, and $100 \mathrm{mM}$ Tris- $\mathrm{HCl}$ buffer). Binding of the aforementioned compounds to BSA at different concentrations ( $0 \%, 0.1 \%, 0.2 \%, 0.5 \%, 1 \%, 1.5 \%$, and $2 \% \mathrm{w} / \mathrm{v})$ in pooled HLMs (at a fixed final microsomal protein concentration of $1 \mathrm{mg} / \mathrm{ml}$ ) was determined via high-throughput equilibrium dialysis, a commonly used technique as previously described by Kariv et al. (2001) and Vanstraelen et al. (2014). After equilibration for 5 hours, solutions from both chambers were removed, quenched, and stored at $-20^{\circ} \mathrm{C}$ until further analysis. The unbound fraction of UGT substrates was assumed to be comparable in the incubation mixture and under the aforementioned conditions in pooled and individual HLMs. Protein binding experiments for trifluoperazine, propofol, amitriptyline, testosterone, and 5-hydroxytryptofol were conducted under contract by Synlab AG (Birsfelden, Switzerland). Protein binding experiments for the remaining substrates were conducted at Hoffmann-La Roche by Florian Klammers.

Effect of BSA on Hepatic UGT Activity. UGT probe substrates were coincubated with BSA at different concentrations $(0 \%, 0.1 \%, 0.2 \%, 0.5 \%, 1 \%$, $1.5 \%$, and $2 \% \mathrm{w} / \mathrm{v}$ ) as well as with optimal incubation buffer and concentrations of $\mathrm{MgCl}_{2}$, UDPGA, and UGT probe substrates in pooled HLMs (at a fixed final microsomal protein concentration of $1 \mathrm{mg} / \mathrm{ml})$. Reactions were terminated after 0.5-30 minutes of incubation, with measurements at eight time points $(0.5,3.5$, $6.5,10,15,20,25$, and 30 minutes). At each BSA concentration, the unbound glucuronidation rate values were obtained by dividing measured total glucuronidation rates by unbound fraction values. In addition, the effect of BSA on the between-subject variability in activity of selected UGT isoforms, for which BSA significantly influences glucuronidation activity, was assessed. Incubations were conducted in the presence of BSA at different concentrations $(0 \%, 0.1 \%$, and $0.5 \% \mathrm{w} / \mathrm{v}$ ) and using 15 individual HLMs derived from single adult donors.

UHPLC-MS/MS Analysis of Glucuronide Metabolites. The UHPLCMS/MS systems used in this study were an LC-30-A series UHPLC system (Shimadzu, Kyoto, Japan) combined with an API 6500 mass spectrometer (AB Sciex LLC, Framingham, MA) and an Nexera X2 autosampler (Shimadzu). Samples $(2 \mu \mathrm{l})$ were injected onto an Ascentis Express C18 HPLC column $(2.7 \mu \mathrm{m}, 2.1 \times 20 \mathrm{~mm}$; Sigma-Aldrich) and eluted with aqueous solution containing $0.1 \%$ formic acid (v/v) (A) and acetonitrile solution containing $0.1 \%$ formic acid $(\mathrm{v} / \mathrm{v})(\mathrm{B})$. The flow rate was set at $0.9 \mathrm{ml} / \mathrm{min}$ over a period of 1 minute. Compounds were eluted using the following gradient conditions: the initial flow rate began at $95 \%$ of solvent $A$ and changed to $2 \%$ at 0.50 minutes, and $95 \%$ at 0.66 minutes to stop at 1.00 minute. Mass detection, ionization mode, and retention time of the investigated compounds are summarized in Table 2. Data acquisition, data handling, and instrument control were performed using the Analyst 1.6 software package (AB Sciex LLC).

To determine the optimal incubation buffer and $\mathrm{MgCl}_{2}$ concentration, the relative UGT activities using specific probe substrates were expressed as the relative rate percentage and assessed by dividing the glucuronide metabolite to internal standard peak area ratio. The peak area ratio obtained in the presence of Tris- $\mathrm{HCl}$ buffer and $10 \mathrm{mM} \mathrm{MgCl}_{2}$ was defined as $100 \%$. To assess the impact of BSA on the microsomal UGT activities, the relative UGT activities using specific probe substrates were expressed as the relative rate percentage and assessed by dividing the glucuronide metabolite to internal standard peak area ratio, with the highest peak area ratio obtained defined as $100 \%$. The results of triplicate incubations are presented as the mean \pm S.D. The parent drug concentration (or relative concentration) was assessed for all incubations to ensure correct spiking of test compounds and no detectable depletion of drug substrate.

Kinetic Data Analysis. Kinetic constants for substrate glucuronidation using HLMs were generated by fitting nonlinear models to experimental data as follows: the Michaelis-Menten equation for a single-enzyme model, as well as the Hill and substrate inhibition equations, as described previously (Hutzler and Tracy, 2002). GraphPad Prism software (version 7.3 for Windows; GraphPad Software, La Jolla, CA) was used. Relative activity corresponding to glucuronide over the internal standard peak area ratio was plotted against UDPGA concentration varying from 1 to $25 \mathrm{mM}$. Visual inspection of fitted functions was used to select the best-fit enzyme kinetic model. The results of triplicate incubations are presented as the mean \pm S.D.

Statistical Analysis. Comparison of the mean glucuronidation rate of UGT isoforms between different experimental conditions and the between-subject

TABLE 2

UHPLC-MS/MS conditions used to determine the glucuronidation activity of hepatic UGT isoforms based on the appearance of metabolite glucuronides of 10 probe substrates in HLMs

\begin{tabular}{|c|c|c|c|c|c|c|}
\hline Analyte & Q1 Mass & Q3 Mass & MRM & Declustering Potential & Collision Energy & Retention Time \\
\hline & \multicolumn{2}{|c|}{$m / z$} & & \multicolumn{2}{|c|}{$V$} & $\min$ \\
\hline$\beta$-Estradiol-3-glucuronide & 447 & 113 & Negative & -55 & -30 & 0.52 \\
\hline Chenodeoxycholic acid-glucuronide & 568 & 382 & Negative & -80 & -50 & 0.46 \\
\hline Trifluoperazine-glucuronide & 584 & 408 & Positive & 75 & 45 & 0.42 \\
\hline 5-Hydroxytryptophol-glucuronide & 352 & 176 & Negative & -30 & -35 & 0.18 \\
\hline Propofol-glucuronide & 353 & 117 & Negative & -40 & -30 & 0.43 \\
\hline Gemfibrozil-glucuronide & 425 & 249 & Negative & -70 & -30 & 0.49 \\
\hline Zidovudine-glucuronide & 442 & 125 & Negative & -80 & -26 & 0.25 \\
\hline Amitriptyline-glucuronide & 454 & 278 & Positive & 80 & 30 & 0.44 \\
\hline Oxazepam-glucuronide ${ }^{a}$ & 464 & 288 & Positive & 80 & 30 & 0.44 \\
\hline Testosterone-glucuronide & 465 & 289 & Positive & 80 & 30 & 0.40 \\
\hline Hexa-deuterated midazolam ${ }^{b}$ & 332 & 297 & Positive & 110 & 25 & 0.37 \\
\hline
\end{tabular}

$\mathrm{m} / \mathrm{z}$, mass-to-charge ratio; MRM, multiple reaction monitoring.

${ }^{a}$ Incubation of racemic oxazepam.

${ }^{b}$ Used as the internal standard. 
variability in the absence and presence of BSA $(0.1 \%$ and $0.5 \% \mathrm{w} / \mathrm{v})$ was performed using a two-tailed $t$ test. A $P$ value $<0.05$ was considered statistically significant. Statistical comparisons were performed using GraphPad Prism software (version 7.3 for Windows).

\section{Results}

Cocktail Incubations of UGT Probe Substrates Using UHPLCMS/MS. We aimed to determine different combinations of UGT probe substrates suitable to measure simultaneously the glucuronidation activity of multiple UGTs in a human hepatic microsomal preparation. For the first time, a set of cocktail assays was developed using the Tecan Fluent system to measure hepatic UGT activity simultaneously for a panel of 10 UGT probe substrates using UHPLC-MS/MS. Four cocktail incubations and one individual incubation ( $\beta$-estradiol) of UGT probe substrates were performed simultaneously within the same run applying similar experimental conditions (Table 3). The simultaneous incubation of two substrates (cocktail 1: trifluoperazine and gemfibrozil; cocktail 2: amitriptyline and oxazepam; and cocktail 3: propofol and zidovudine) or three substrates (cocktail 4: chenodeoxycholic acid, 5-hydroxytryptophol, and testosterone) revealed no changes in glucuronidation activity $(<1.2$-fold) compared with individual incubations. In addition, no metabolic or analytical interactions among selected UGT probe substrates were detected for any metabolites during cocktail incubations in pooled HLMs. The relative S.D. values ranged from $4.4 \%$ to $24.0 \%$ for the results of the cocktail incubation procedure, which were comparable to those of the individual incubations $(3.3 \%-19.6 \%)$.

Optimal Incubation Buffer and $\mathbf{M g C l}_{2}$ Concentration. A panel of 10 selective UGT probe substrates was incubated to determine the glucuronidation activity of hepatic UGT isoforms in alamethicinpretreated pooled HLMs. The effect of incubation buffer (i.e., Tris$\mathrm{HCl}$ and potassium phosphate buffers, as well as increasing $\mathrm{MgCl}_{2}$ concentrations $(0,1,2,5,8$, and $10 \mathrm{mM})$ was assessed on the glucuronidation activity of major hepatic UGT isoforms. Incubation of UGT probe substrates showed that the rate of glucuronide formation in pooled HLMs (1 mg/ml) was linear up to 3.5 minutes. A fixed microsomal protein concentration of $1 \mathrm{mg} / \mathrm{ml}$ was selected to represent high enzyme activity conditions under which most (relatively stable) candidate drugs are likely to be assessed and where monitoring the UGT enzymes is subject to the highest challenge in terms of probe substrate metabolism linearity. For amitriptyline (UGT2B10) and oxazepam (UGT2B15), incubation time data up to 15 minutes were used. These conditions were applied in subsequent experiments. All glucuronidation activity values were expressed as the relative rate percentage (i.e., peak area ratio of glucuronide over the internal standard) and normalized with the ratio obtained using Tris- $\mathrm{HCl}$ buffer in the presence of $10 \mathrm{mM}$ $\mathrm{MgCl}_{2}$, which was defined as $100 \%$.

Incubations of pooled HLMs formed the glucuronides of all selected UGT substrates in the absence and presence of $\mathrm{MgCl}_{2}$, and with both incubation buffers (Fig. 1). The glucuronidation rates increased with increasing $\mathrm{MgCl}_{2}$ concentrations with either Tris- $\mathrm{HCl}$ or potassium phosphate incubation buffers. The greatest glucuronidation rates were observed in the presence of $10 \mathrm{mM} \mathrm{MgCl}_{2}$, with the exception of oxazepam (UGT2B15) and testosterone (UGT2B17), for which the maximum glucuronidation activity was obtained at 5 and $8 \mathrm{mM} \mathrm{MgCl}_{2}$, respectively. However, the $10 \mathrm{mM}$ condition was less than $10 \%$ lower than the maximum activity observed for these substrates. In Tris- $\mathrm{HCl}$ incubations and in the absence of $\mathrm{MgCl}_{2}$, the activity of UGT1A9 (propofol) and UGT1A6 (5-hydroxytryptophol) represented $13 \%$ and $74 \%$ of the activity obtained in the presence of $10 \mathrm{mM} \mathrm{MgCl}$, respectively.

In the presence of $10 \mathrm{mM} \mathrm{MgCl}$, glucuronidation activities were significantly greater with Tris-HCl buffer compared with potassium phosphate buffer for chenodeoxycholic acid (UGT1A3), trifluoperazine (UGT1A4), propofol (UGT1A9), gemfibrozil (UGT2B4/2B7), and testosterone (UGT2B17) (Fig. 1). In contrast, no significant differences in glucuronidation rates were detected for $\beta$-estradiol (UGT1A1), 5-hydroxytryptophol (UGT1A6), zidovudine (UGT2B7), amitriptyline (UGT2B10), and oxazepam (UGT2B15). Interestingly, a smaller coefficient of variation was associated with the glucuronidation activities measured with Tris- $\mathrm{HCl}$ buffer compared with potassium phosphate buffer, although the reason for this observation is not currently known.

Optimal Concentration of UDPGA. Incubations of UGT probe substrates were conducted using pooled HLMs with Tris- $\mathrm{HCl}$ buffer, $10 \mathrm{mM} \mathrm{MgCl}_{2}$, and a UDPGA concentration ranging from 1 to $25 \mathrm{mM}$. Kinetic profiling revealed that the formation of $\beta$-estradiol3-glucuronide (UGT1A1), propofol-glucuronide (UGT1A9), and zidovudine-glucuronide (UGT2B7) followed hyperbolic MichaelisMenten kinetics, as illustrated in Fig. 2. However, the formation of glucuronides of chenodeoxycholic acid (UGT1A3), trifluoperazine (UGT1A4), amitriptyline (UGT2B10), oxazepam (UGT2B15), and testosterone (UGT2B17) exhibited sigmoidal kinetics and was modeled by the Hill equation. When coincubated with $5 \mathrm{mM}$ UDPGA, the glucuronidation activity of those UGT isoforms represented typically

TABLE 3

Cocktail incubation approach using pooled HLMs and the Tecan Fluent system Ten UGT probe substrates were incubated either individually or in cocktails. Values are presented as the mean \pm S.D. unless otherwise mentioned.

\begin{tabular}{|c|c|c|c|c|c|c|}
\hline \multirow{2}{*}{ Cocktail Group } & \multirow{2}{*}{ UGT Probe Substrate } & \multirow{2}{*}{ UGT Isoform } & \multirow{2}{*}{ Incubation Concentration } & \multicolumn{2}{|c|}{ Relative Rate $(\mathrm{CV})^{b}$} & \multirow{2}{*}{ Fold Difference } \\
\hline & & & & Individual Incubation & Cocktail Incubation & \\
\hline & & & $\mu M$ & $\%$ & & \\
\hline \multirow[t]{2}{*}{1} & Trifluoperazine & UGT1A4 & 5 & $100 \pm 3.3$ & $111.5 \pm 7.3$ & 1.1 \\
\hline & Gemfibrozil & $\mathrm{UGT} 2 \mathrm{~B} 4 / 2 \mathrm{~B} 7$ & 5 & $100 \pm 12.9$ & $92.1 \pm 13.6$ & 0.9 \\
\hline \multirow[t]{2}{*}{2} & Amitriptyline & UGT2B10 & 5 & $100 \pm 10.7$ & $94.2 \pm 20.0$ & 0.9 \\
\hline & Oxazepam $^{a}$ & UGT2B15 & 5 & $100 \pm 2.0$ & $104.7 \pm 21.2$ & 1.0 \\
\hline \multirow[t]{2}{*}{3} & Propofol & UGT1A9 & 5 & $100 \pm 13.3$ & $105.1 \pm 11.8$ & 1.1 \\
\hline & Zidovudine & UGT2B7 & 100 & $100 \pm 4.4$ & $97.5 \pm 4.4$ & 1.0 \\
\hline \multirow[t]{3}{*}{4} & Chenodeoxycholic acid & UGT1A3 & 5 & $100 \pm 10.9$ & $98.0 \pm 7.6$ & 1.0 \\
\hline & 5-Hydroxytryptophol & UGT1A6 & 5 & $100 \pm 12.0$ & $101.0 \pm 6.1$ & 1.0 \\
\hline & Testosterone & UGT2B17 & 5 & $100 \pm 3.9$ & $87.1 \pm 7.2$ & 0.9 \\
\hline 5 & $\beta$-Estradiol & UGT1A1 & 5 & N/A & N/A & N/A \\
\hline
\end{tabular}

$\mathrm{CV}$, coefficient of variation; N/A, not applicable.

${ }^{a}$ Incubation of racemic oxazepam.

${ }^{b}$ Data are reported as the relative rate percentage $(\mathrm{CV} \%)(n=3)$. The relative rate was measured in the individual incubation and was defined as $100 \%$ for each substrate. 
A Estradiol (3-Gluc) (UGT1A1)

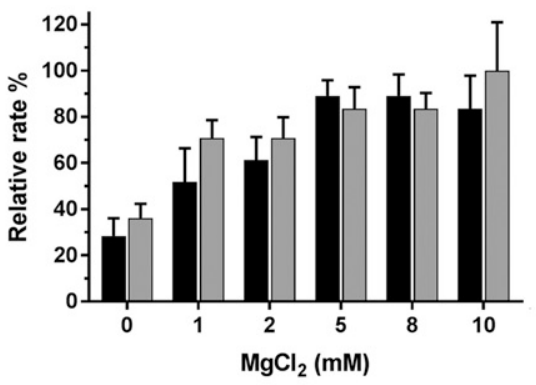

D

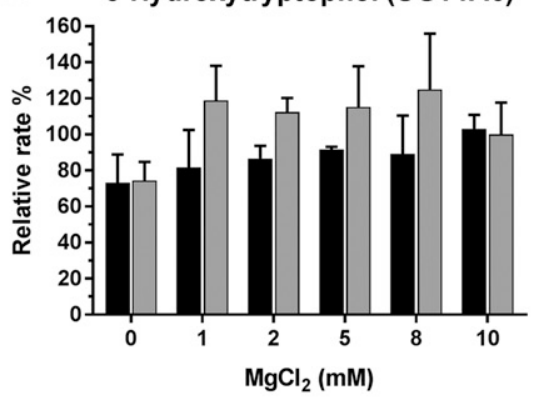

G

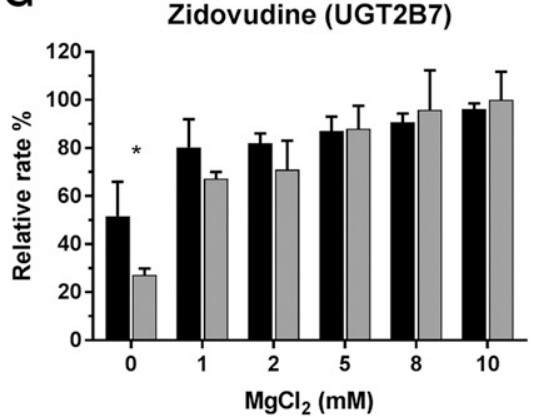

J

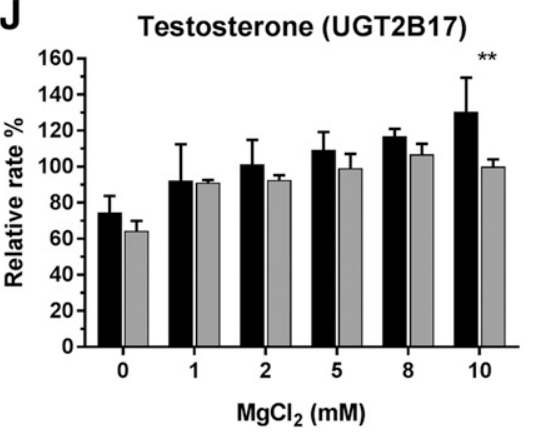

B

Chenodeoxycholic acid (UGT1A3)

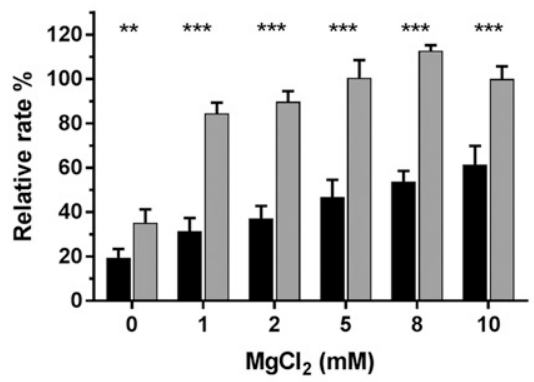

E

Propofol (UGT1A9)

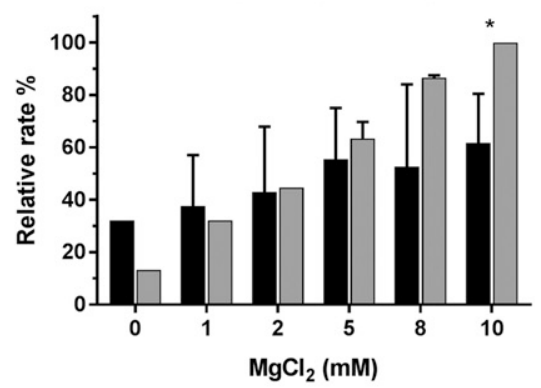

H

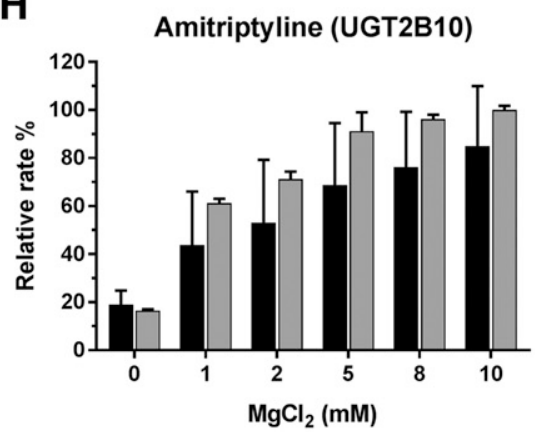

C

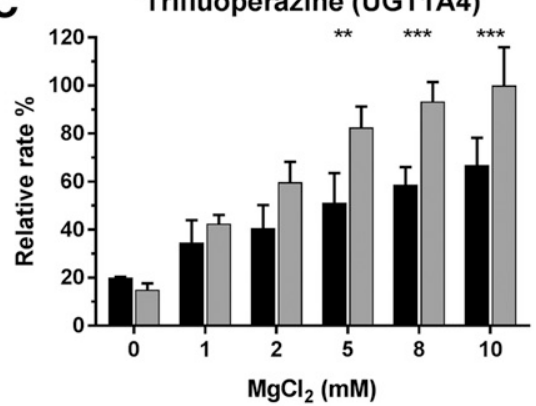

F

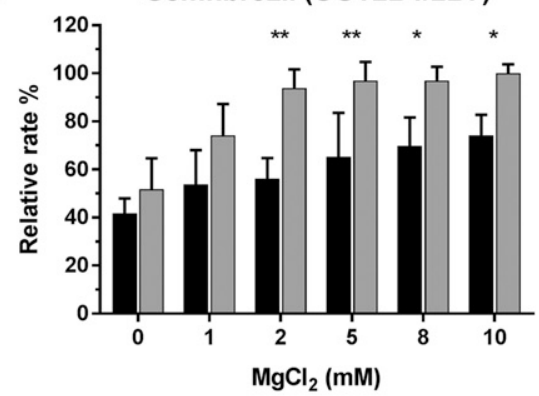

I

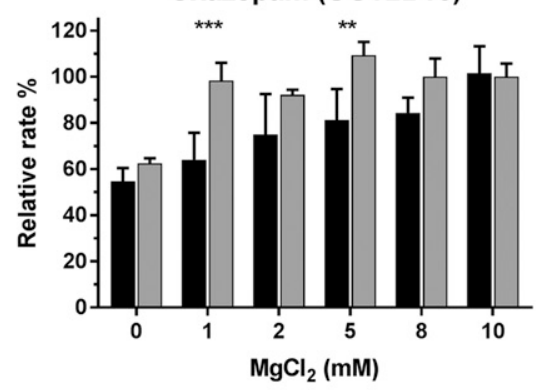

Fig. 1. Effect of incubation buffer and increasing $\mathrm{MgCl}_{2}$ concentrations on hepatic UGT activities in pooled HLMs. Bars represent the relative glucuronidation rates of UGT isoforms determined using estradiol (A), chenodeoxycholic acid (B), trifluoperazine (C), 5-hydroxytryptophol (D), propofol (E), gemfibrozil (F), zidovudine (G), amitriptyline $(\mathrm{H})$, oxazepam (I), and testosterone (J) in the absence and presence of $\mathrm{MgCl}_{2}$ at different concentrations $(1,2,5,8$, and $10 \mathrm{mM})$, and with either potassium phosphate buffer (black) or Tris-HCl buffer (gray) $(100 \mathrm{mM}, \mathrm{pH} 7.4)$. For each substrate, the relative UGT activities were expressed as the relative rate percentage and were assessed by dividing the glucuronide metabolite to internal standard peak area ratio, with the peak area ratio obtained in the presence of Tris- $\mathrm{HCl}$ buffer and $10 \mathrm{mM} \mathrm{MgCl}{ }_{2}$ defined as $100 \%$. $* P<0.05 ; * * P<0.01 ; * * * P<0.001$ (paired $t$ test between phosphate and Tris-HCl incubation buffers). Each bar represents mean \pm S.D. $(n=3$ ).

more than $65 \%$ of the maximal activity, with the exception of amitriptyline (52\%). However, for some compounds, the maximal velocity was not fully reached at the $25 \mathrm{mM}$ concentration of UDPGA, and the formation rate of trifluoperazine-glucuronide did not reach a plateau at high UDPGA concentrations; UGT1A4 activity represented only $47 \%$ of maximal activity at $5 \mathrm{mM}$ UDPGA. Chenodeoxycholic acid, trifluoperazine, and testosterone glucuronidation, via UGT1A3, UGT1A4, and UGT2B17, respectively, exhibited positive cooperativity in cofactor binding. The detailed kinetic parameters are summarized in Table 4.

The formation of 5-hydroxytryptophol-glucuronide (UGT1A6) and gemfibrozil-glucuronide (UGT2B4/2B7) displayed substrate inhibition kinetics when coincubated with increasing UDPGA concentrations. In contrast to other UGT probe substrates, the glucuronidation rates started to decrease above concentrations of 3.75-5 mM UDPGA. At 8 and $25 \mathrm{mM}$ UDPGA, a $21 \%$ and $46 \%$ reduction in UGT1A6 activity was 
A

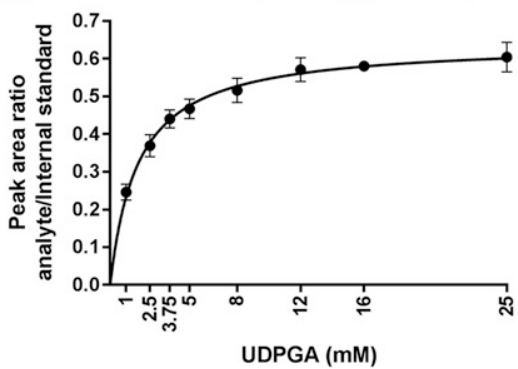

D Chenodeoxycholic acid (UGT1A3)

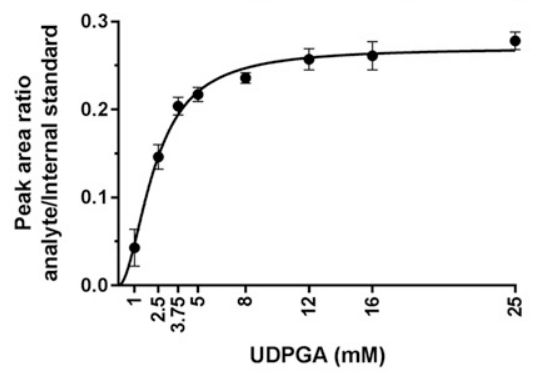

G

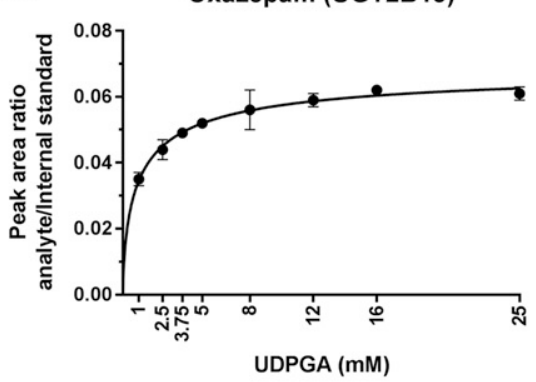

\section{$\mathbf{J}$}

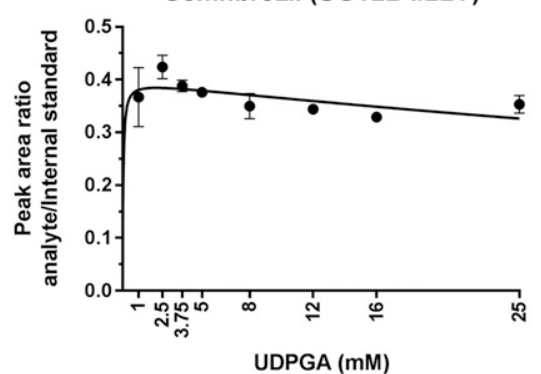

B

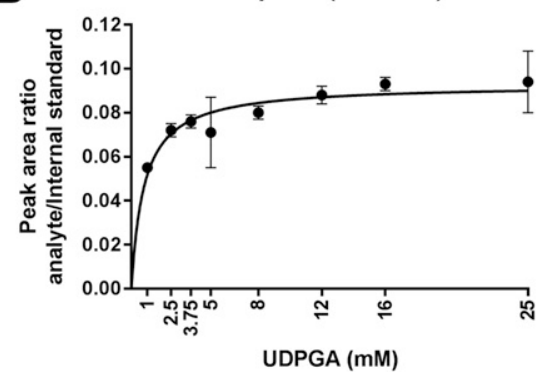

E

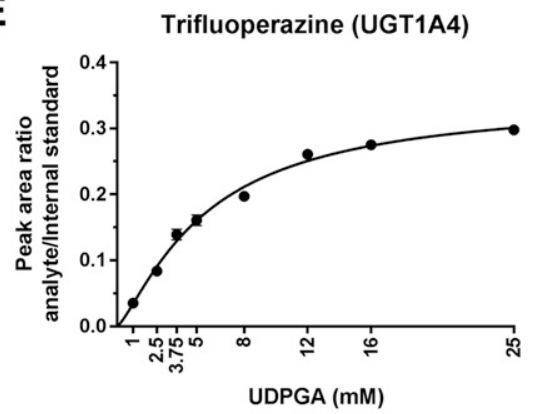

H

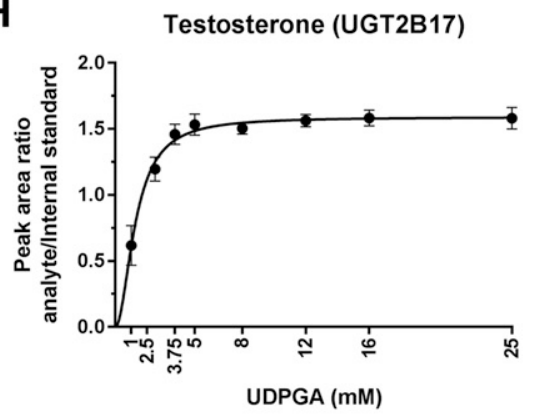

K

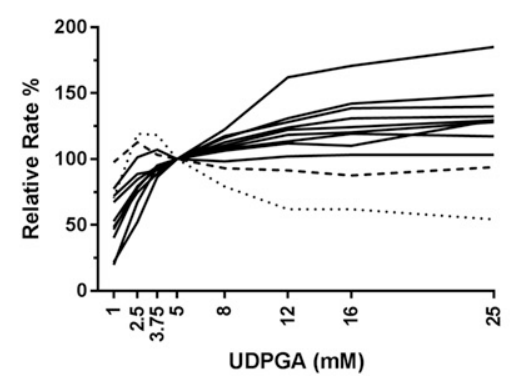

C

Zidovudine (UGT2B7)

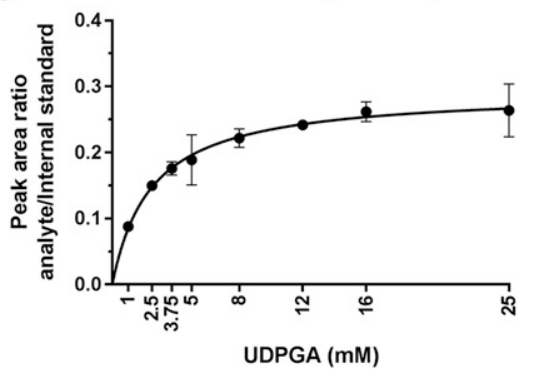

$\mathbf{F}$

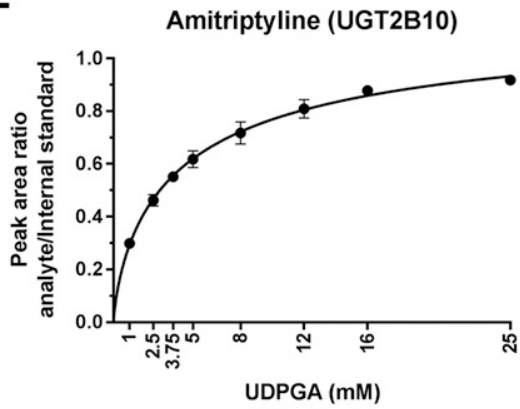

I

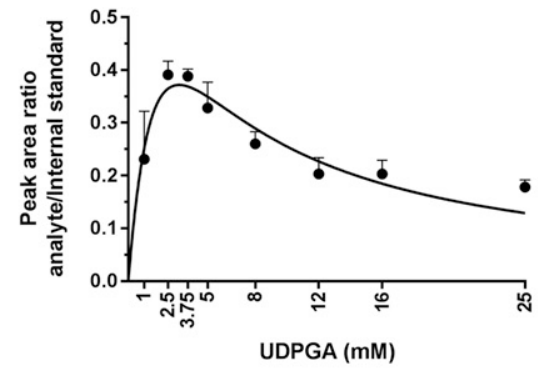

Fig. 2. Kinetics of 10 UGT probe substrates over a wide UDPGA concentration range $(1,2.5,3.75,5,8,12,16$, and $25 \mathrm{mM})$ in pooled HLMs. Symbols represent the mean peak area ratio of glucuronide metabolite to internal standard \pm S.D. $(n=3)$. The solid line represents the fit of the reaction velocity plotted against increasing UDPGA concentrations using nonlinear least-squares regression analysis. Experimental data were fitted by using a Michaelis-Menten kinetic model estradiol (A), propofol (B) and zidovudine (C), an allometric sigmoidal kinetic model chenodeoxycholic acid (D), trifluoperazine (E), amitriptyline (F), oxazepam (G), and testosterone $(\mathrm{H})$, and a substrate inhibition kinetic model 5-hydroxytryptophol (I) and gemfibrozil (J) Relative glucuronidation rate of UGT isoforms using selective UGT probe substrates over a wide UDPGA concentration range $(1,2.5,3.75,5,8,12,16$, and $25 \mathrm{mM})$ in pooled HLMs (K). Relative UGT activities were expressed as the relative rate percentage and were assessed by dividing the glucuronide metabolite to internal standard peak area ratio, with the peak area ratio obtained in the presence of $5 \mathrm{mM}$ UDPGA defined as $100 \%$. Dotted and dashed lines represent the relative glucuronidation formation of 5-hydroxytryptophol (UGT1A6) and gemfibrozil (UGT2B4/2B7).

observed compared with that at $5 \mathrm{mM}$ UDPGA, respectively (Fig. 2K). Similarly, a $6 \%-12.5 \%$ reduction in gemfibrozil-glucuronide formation was observed above a concentration of $5 \mathrm{mM}$ UDPGA. As a result, $5 \mathrm{mM}$ was defined as an optimal concentration of UDPGA to measure simultaneously the microsomal glucuronidation activity of hepatic UGT isoforms in an automated glucuronidation assay while also ensuring that reagent costs are not prohibitive.
Effect of BSA on Hepatic Activity of UGT Isoforms. This study clearly demonstrated that the effect of BSA supplementation on hepatic glucuronidation rates was enzyme and substrate dependent (Fig. 3). The total glucuronidation rate of UGT1A9 and UGT2B7, using propofol and zidovudine as probe substrates, increased by 1.6- and 3.4-fold, respectively, with $2 \%$ BSA supplementation. However, compared with the absence of BSA, the total metabolic activity of UGT1A4 (trifluoperazine 
TABLE 4

Hepatic glucuronidation activities using 10 UGT probe substrates in pooled HLMs and in the presence of increasing UDPGA concentrations (1, 2.5, 3.75, 5, 8, 12, 16, and $25 \mathrm{mM}$ )

Values are presented as the mean \pm S.D. unless otherwise mentioned.

\begin{tabular}{|c|c|c|c|c|c|c|c|}
\hline UGT Substrate Concentration $(5 \mu \mathrm{M})$ & $K_{\mathrm{m}}$ & Maximal Reaction Rate & $\mathrm{S}_{50}$ & Hill Coefficient & $K_{\mathrm{i}}$ & $\begin{array}{l}\text { Maximal Activity at } \\
5 \mathrm{mM} \text { UDPGA }\end{array}$ & Model \\
\hline & $m M$ & & $m M$ & & $m M$ & $\%$ & \\
\hline$\beta$-Estradiol & $1.77 \pm 0.14$ & $0.64 \pm 0.01$ & N/A & N/A & N/A & 73 & MM \\
\hline Chenodeoxycholic acid & $4.79 \pm 0.80$ & $0.27 \pm 0.01$ & $2.29 \pm 0.12$ & $1.89 \pm 0.18$ & N/A & 80 & SMM \\
\hline Trifluoperazine & $9.13 \pm 0.99$ & $0.34 \pm 0.02$ & $5.59 \pm 0.56$ & $1.29 \pm 0.11$ & N/A & 47 & SMM \\
\hline 5-Hydroxytryptophol & $2.79 \pm 1.74$ & $1.02 \pm 0.40$ & N/A & N/A & $3.69 \pm 2.02$ & $84^{c}$ & SI \\
\hline Propofol & $0.80 \pm 0.17$ & $0.09 \pm 0.00$ & N/A & N/A & N/A & 76 & MM \\
\hline Gemfibrozil & $0.04 \pm 0.10$ & $0.40 \pm 0.02$ & N/A & N/A & $113.7 \pm 61.8$ & $89^{c}$ & SI \\
\hline Zidovudine $^{a}$ & $2.47 \pm 0.33$ & $0.29 \pm 0.01$ & N/A & N/A & N/A & 65 & MM \\
\hline Amitriptyline & $3.07 \pm 0.19$ & $1.19 \pm 0.07$ & $4.47 \pm 0.83$ & $0.75 \pm 0.07$ & N/A & 52 & SMM \\
\hline Oxazepam ${ }^{b}$ & $1.02 \pm 0.12$ & $0.07 \pm 0.00$ & $1.04 \pm 0.18$ & $0.68 \pm 0.14$ & N/A & 75 & SMM \\
\hline Testosterone & $1.63 \pm 0.20$ & $1.59 \pm 0.03$ & $1.28 \pm 0.07$ & $1.94 \pm 0.21$ & N/A & 96 & SMM \\
\hline
\end{tabular}

$K_{\mathrm{i}}$, substrate inhibition constant; $K_{\mathrm{m}}$, UDPGA affinity constant; MM, hyperbolic Michaelis-Menten model; N/A, not available; $\mathrm{S}_{50}$, UDPGA concentration at which half of maximal activity is reached; SI, substrate inhibition model; SMM, sigmoid Michaelis-Menten model.

${ }^{a}$ Incubation of $100 \mu \mathrm{M}$ zidovudine.

${ }^{b}$ Incubation of racemic oxazepam.

${ }^{c}$ Maximal activity reached at $2.5 \mathrm{mM}$ UDPGA.

glucuronidation) in HLMs decreased by $12.5 \%$ in the presence of $2 \%$ BSA (Fig. 3, part I). For the remaining compounds, the metabolic activity of UGT1A1, UGT1A3, UGT2B15, and UGT2B17 decreased by $75 \%, 91 \%$, $87 \%$, and $60 \%$ with the $2 \%$ BSA supplementation, respectively. The total glucuronidation activity of UGT1A6 (5-hydroxytryptophol glucuronidation) and UGT2B10 (amitriptyline glucuronidation) remained unchanged with increasing BSA concentrations.

In the absence of BSA, the unbound fraction of UGT probe substrates ranged from less than $2 \%$ for trifluoperazine to $100 \%$ for zidovudine and 5-hydroxytryptophol (Fig. 3, part I). The effect of BSA on the unbound fraction varied between substrates. No changes in unbound fraction were observed for trifluoperazine, propofol, and amitriptyline with increasing BSA concentrations up to $2 \%$. The unbound fraction for gemfibrozil reached values near the lower limit of quantification with increasing BSA concentrations. In contrast, for the remaining probe substrates, the fraction unbound measured in the incubation mixture decreased in the presence of BSA, between 1.5-fold and 6.3-fold for 5-hydroxytryptophol and chenodeoxycholic acid, respectively. However, unbound fractions could not be determined for $\beta$-estradiol with increasing BSA concentrations.

Glucuronide formation rates of UGT substrates were corrected for the experimentally measured unbound fraction (Fig. 3, part II). Our results indicated that the optimal BSA concentration at which the greatest unbound glucuronidation activity was obtained differed across UGT isoforms, being both enzyme and substrate dependent. With the exception of UGT1A6, the greatest unbound glucuronidation rates were not systematically obtained in the presence of $2 \%$ BSA. The greatest unbound glucuronidation activities were obtained in the presence of $0.2 \%$ BSA (testosterone via UGT2B17), $0.5 \%$ BSA (chenodeoxycholic acid via UGT1A3), and 1\% BSA (propofol via UGT1A9, zidovudine via UGT2B7, and oxazepam via UGT2B15), compared with those without BSA supplementation. In addition, BSA inhibitory effects on unbound glucuronidation activities were observed for several UGT isoforms from $0.1 \%$ BSA for UGT1A4, 0.5\% BSA for UGT2B17, or $1 \%$ BSA for UGT1A3, UGT1A9, UGT2B7, and UGT2B15.

Effect of BSA on Between-Subject Activity of UGT Isoforms. The effect of BSA on the between-subject variability in the formation rate of chenodeoxycholic acid-glucuronide (UGT1A3), trifluoperazineglucuronide (UGT1A4), propofol-glucuronide (UGT1A9), and zidovudine-glucuronide (UGT2B7) was assessed using 15 individual HLMs characterized with low, moderate, and high corresponding glucuronidation activity (in-house data). As illustrated in Fig. 4, the between-subject variability in glucuronidation activity was not significantly reduced in the presence of either $0.1 \%$ or $0.5 \%$ BSA compared with that to $0 \%$ BSA $(P>0.05)$. Between-subject variability of zidovudine-glucuronide formation (UGT2B7) decreased up to $12 \%$ in the presence of $0.5 \%$ BSA. In contrast, the variability in UGT1A9 activity (propofol-glucuronide) across individuals slightly increased by $5 \%$.

\section{Discussion}

In our study, we aimed to develop and optimize an automated UHPLC-MS/MS-based UGT profiling assay to assess simultaneously the glucuronidation activity of 10 hepatic UGT isoforms using a cocktail incubation approach in HLMs. We identified and implemented methodological and technical improvements to reduce the discrepancies in glucuronidation activity observed across studies, which are in part due to a lack of standardization (Badée et al., 2018).

We developed an automated assay using a Tecan Fluent system at Hoffman-La Roche Ltd. Due to its flexible and configurable architecture, a large number of experimental condition combinations could be achieved and this facilitated the systematic testing that is required to improve the measurement of in vitro glucuronidation activity. Our proposed UGT profiling assay is intended for UGT clearance optimization and phenotyping. Prediction of human drug clearance by in vitro to in vivo scaling data has not been the objective.

Cocktails and individual incubations of selected UGT probe substrates using HLMs were compared to ensure that interactions among substrates were avoided. The proposed substrate cassetting approach allows efficient monitoring of 10 glucuronidation activities with minimal effect on enzyme activity or analytical interactions among substrates even under high metabolism conditions (Table 3). However, compared with the situation for cytochrome P450 enzymes where selective substrates and inhibitors exist (Zientek and Youdim, 2015), UGT isoform-selective substrates or inhibitors are either limited or not available. Our study highlighted the critical challenge of obtaining selective UGT probe substrates. Although some of the UGT probe substrate reactions used in this study show good enzyme selectivity (e.g., for $\beta$-estradiol, trifluoperazine, 5-hydroxytryptophol, propofol, and zidovudine), other probe substrates such as gemfibrozil, oxazepam, and chenodeoxycholic acid are less selective. Gemfibrozil is metabolized by both UGT2B4 and UGT2B7 isoforms (Mano et al., 2007). In these studies, no selective UGT2B7 inhibitor was coincubated with gemfibrozil, but this could be 
$I_{A}$

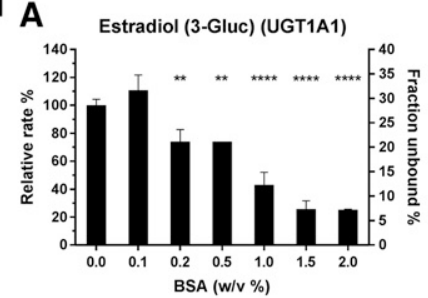

E

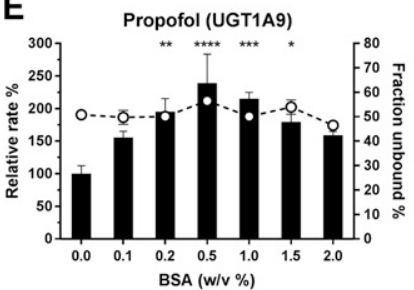

I

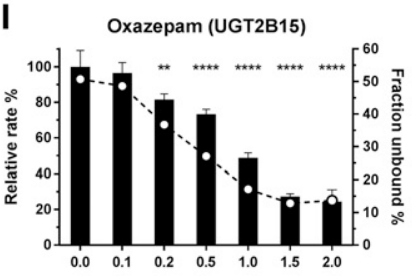

II

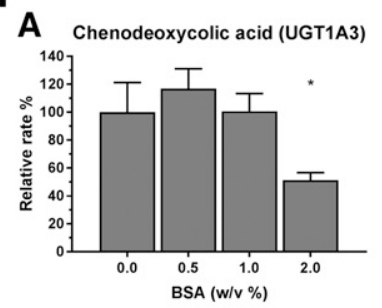

E

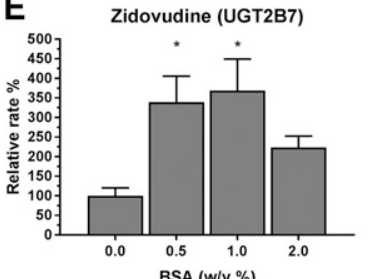

B

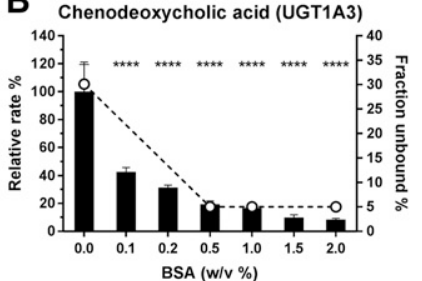

F Gemfibrozil (UGT2B4/2B7)
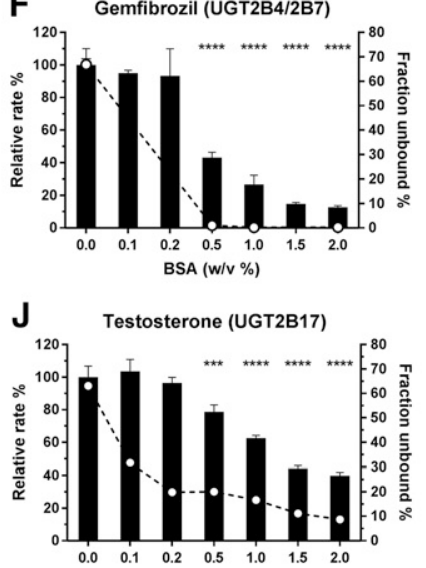

BSA (w/v \%)

B

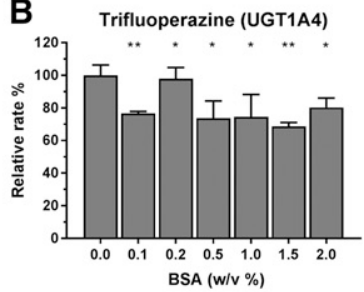

F

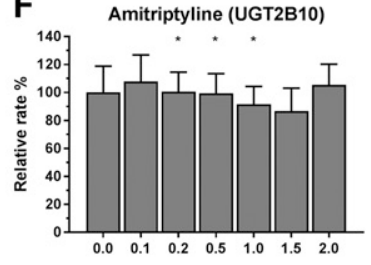

C
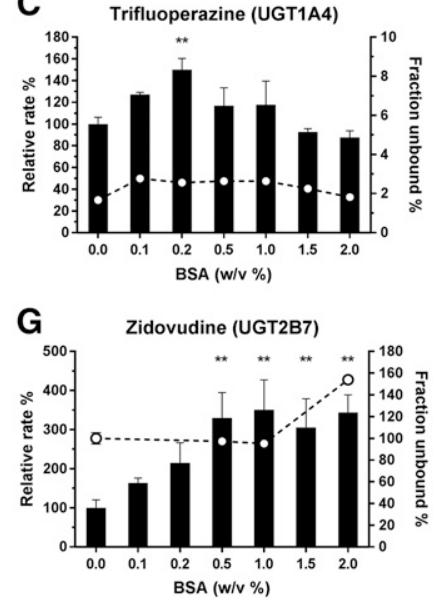

H
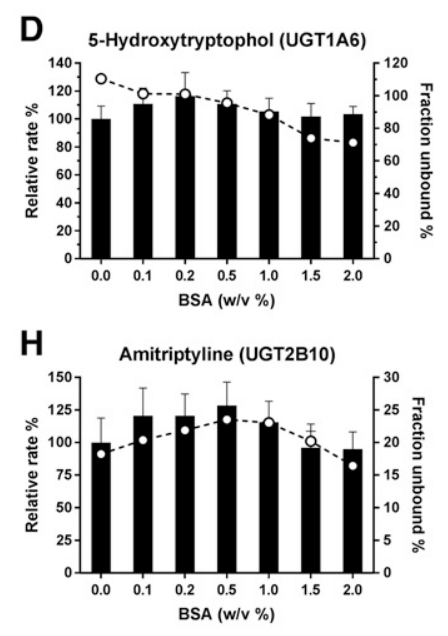

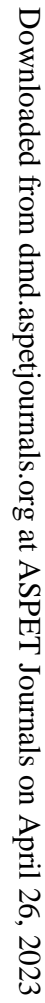

Fig. 3. Effect of increasing BSA concentrations on the total glucuronidation rates of hepatic UGT isoforms in pooled HLMs (part I). Circles and dashed lines represent the unbound fraction of estradiol (A), chenodeoxycholic acid (B), trifluoperazine (C), 5-hydroxytryptophol (D), propofol (E), gemfibrozil (F), zidovudine $(\mathrm{G})$, amitripyline $(\mathrm{H})$, oxazepam $(\mathrm{I})$, and testosterone $(\mathrm{J})$ measured in HLMs in the absence and presence of increasing BSA concentrations $(0.1 \%, 0.2 \%, 0.5 \%, 1 \%$, $1.5 \%$, and $2 \% \mathrm{w} / \mathrm{v}$ ). Black bars represent the total relative rate percentage, which were determined by dividing the glucuronide metabolite to internal standard peak area ratio. Effect of increasing BSA concentrations on the unbound glucuronidation rates of hepatic UGT isoforms in pooled HLMs (part II). Protein binding was determined via high-throughput equilibrium dialysis for chenodeoxycholic acid (A), trifluoperazine (B), 5-hydroxytryptophol (C), propofol (D), zidovudine (E), amitriptyline $(\mathrm{F})$, oxazepam $(\mathrm{G})$, and testosterone $(\mathrm{H})$. Protein binding in HLMs was not measurable for $\beta$-estradiol. Gray bars represent the unbound glucuronidation relative rates corrected by the experimentally measured unbound fraction. For each substrate, the glucuronide metabolite to internal standard peak area ratio obtained without BSA supplementation was defined as $100 \%$. ${ }^{*} P<0.05 ; * * P<0.01$; ${ }^{* * *} P<0.001$ (paired $t$ test compared with $0 \%$ BSA). Each bar represents mean \pm S.D. $(n=3$ ).

done in the future to enhance the selectivity of gemfibrozil for UGT2B4. In addition, oxazepam was formulated as a racemic preparation of $S$ - and $R$ enantiomers, as $S$-oxazepam was not available. $S$-oxazepam is principally metabolized by UGT2B 15 , whereas $R$-oxazepam is also metabolized by additional UGTs, mainly UGT1A9 and UGT2B7 (Court et al., 2002). However, we demonstrated in stereoselective analyses that $S$-oxazepam glucuronide was mostly generated by pooled HLMs $(80.7 \% \pm 2.9 \%)$ compared with $R$-oxazepam glucuronide under the incubation conditions used in this study (in-house data), which allowed the use of the racemic oxazepam to assess the microsomal glucuronidation activity of UGT2B15. Chenodeoxycholic acid is mainly metabolized by UGT1A3, with a minor relative contribution of UGT2B7 and UGT1A1 (Gradinaru et al., 2015). The relative activity of recombinantly expressed UGT1A1 and UGT2B7 isoforms was less than $1.5 \%$ of UGT1A3 activity in chenodeoxycholic acid glucuronidation (in-house data), indicating a good selectivity of chenodeoxycholic acid for UGT1A3. However, chenodeoxycholic acid would not be a useful UGT1A3 probe substrate in a more complex system such as hepatocytes, due to very high taurine and glycine conjugation rates (Thakare et al., 2018). Raloxifene, considered as a partial substrate of UGT1A8 and UGT1A10 (Kemp et al., 2002), was not included in our report, as neither UGT isoform was detected in HLMs (Fallon et al., 2013; Margaillan et al., 2015). However, we found that raloxifene could be 
A

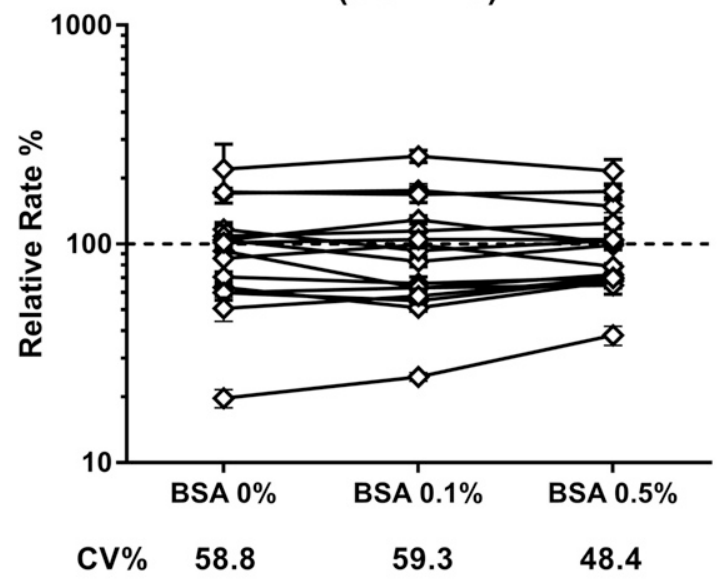

C

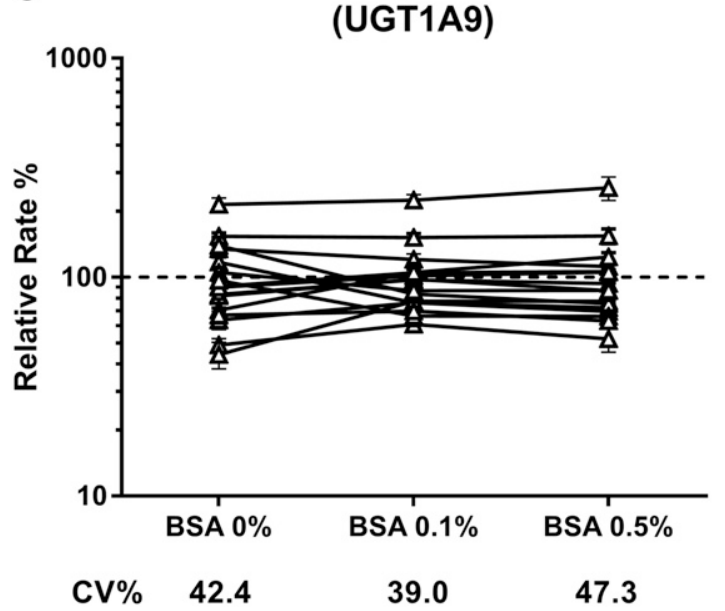

B 5-Hydroxytryptophol
(UGT1A6)

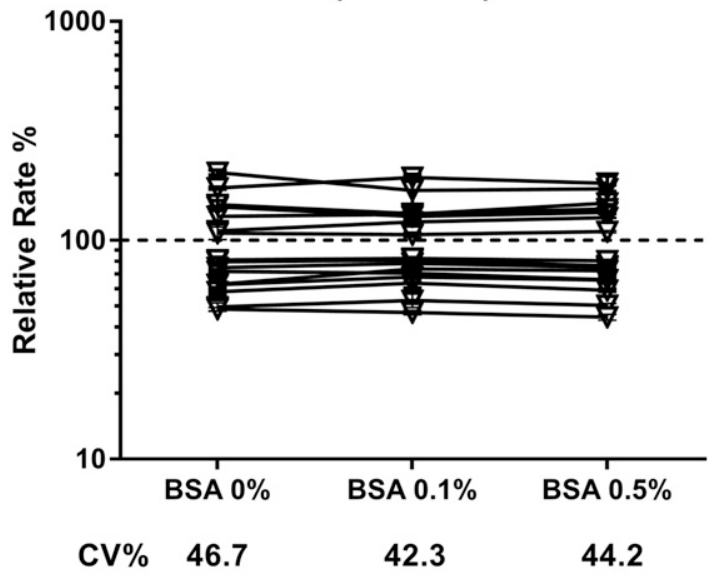

D
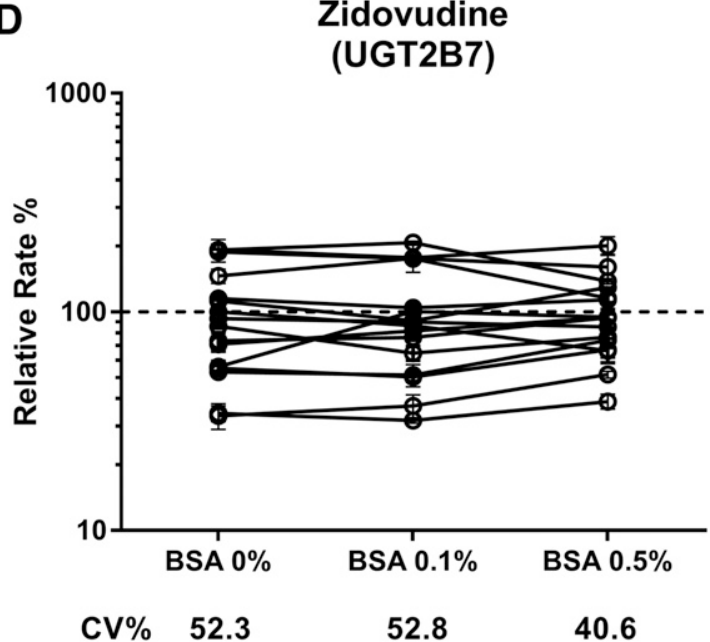

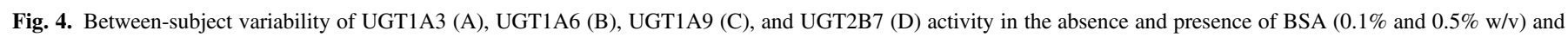

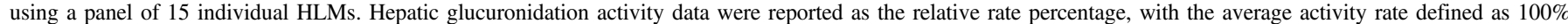
(dashed line). Each point represents mean \pm S.D. $(n=3)$. CV, coefficient of variation.

combined with trifluoperazine and gemfibrozil in a cassette (at $5 \mu \mathrm{M}$ ) (inhouse data) and may be a useful additional probe for investigations of extrahepatic raloxifene metabolism using human intestinal microsomes, where UGT1A8 and UGT1A10 are highly expressed (Tukey and Strassburg, 2000).

The lack of standardization of incubation conditions of in vitro glucuronidation assays is one of the main sources of interstudy variability. Therefore, we examined whether standardized incubation conditions would enhance the determination of glucuronide formation rates of multiple hepatic UGT isoforms in HLMs. Our results indicated that greater microsomal glucuronidation activity by different hepatic UGT isoforms was obtained using $10 \mathrm{mM} \mathrm{MgCl} 2$ and $5 \mathrm{mM}$ UDPGA with $100 \mathrm{mM}$ Tris- $\mathrm{HCl}$ buffer. This is in agreement with a previous study by Walsky and colleagues. They demonstrated greater activity of five hepatic UGT isoforms (UGT1A1, UGT1A4, UGT1A6, UGT1A9, and UGT2B7) using $10 \mathrm{mM} \mathrm{MgCl}_{2}$ with $100 \mathrm{mM}$ Tris- $\mathrm{HCl}$ buffer compared with those obtained with potassium phosphate buffer and 0-5 $\mathrm{mM} \mathrm{MgCl}_{2}$. Our results further revealed that glucuronidation activities of previously less characterized UGT isoforms (UGT1A3, UGT2B10, UGT2B15, and UGT2B17) were enhanced under the aforementioned optimized conditions (Fig. 1).
A $5 \mathrm{mM}$ UDPGA concentration is generally used in UGT phenotyping assays in HLMs, although the impact of varying UDPGA concentration has not yet been systematically examined. A recent publication from Lu et al. (2017) demonstrated that the maximal metabolic activity of UGT1A4 and UGT2B10 was reached when $8 \mathrm{mM}$ UDPGA was used, which was the highest UDPGA concentration tested. In this study, we demonstrated that the microsomal activity of hepatic UGT isoforms increased as the UDPGA concentration increased. The selected substrates panel, with the exception of 5-hydroxytryptophol and gemfibrozil, followed either hyperbolic Michaelis-Menten kinetics or sigmoidal kinetics, reaching $52 \%-96 \%$ of maximal activity at $5 \mathrm{mM}$ UDPGA (Fig. 2). Thus, we demonstrated that the concentration of the main cosubstrate had a significant impact on the microsomal UGT metabolic capacity. The reasons why the activity of UGT1A6 and UGT2B4/2B7 decreases with UDPGA concentrations above $5 \mathrm{mM}$ remain to be determined, but a recent article by Walia et al. (2018) supports our findings. They found that the combination of 5 mM UDPGA and $10 \mathrm{mM} \mathrm{MgCl}$ led to maximal activities in both HLMs and recombinantly expressed UGT1A1 and UGT1A4 isoforms, but not in UGT1A6 and UGT1A9, for which the specific mechanism appeared to be susceptibility to inhibition by UDP (a product of the cosubstrate). 
Of note, saccharolactone was not included in this optimization. Walsky et al. (2012) demonstrated that saccharolactone supplementation did not significantly affect the microsomal activity of UGT1A1, UGT1A6, and UGT2B7, whereas decreased activity of UGT1A4 and UGT1A9 was observed. Nevertheless, inclusion of saccharolactone is favored by some laboratories and its addition may be helpful where significant microsomal glucuronidase activity toward individual drug compounds exists.

The inclusion of BSA is thought to sequester inhibitory fatty acids produced during microsomal incubation, and enhanced the turnover of some UGT substrates while inhibiting that of others (Rowland et al., 2007, 2008; Walsky et al., 2012). Our results are consistent with published data. Total and unbound UGT1A9 and UGT2B7 glucuronidation activity, using propofol and zidovudine as probe substrates, increased by up to $74 \%$ and $343 \%$, respectively, in the presence of $2 \%$ BSA compared with that in the absence of BSA (Fig. 3). Although the addition of BSA enhanced the unbound glucuronidation activity of a number of UGT isoforms, the recommended BSA concentration of $2 \%$ did not systematically produce the greatest unbound activity. We demonstrated that the BSA inhibitory effects on either total or unbound glucuronidation activities of UGTs were BSA concentration, isoform, and substrate dependent. We noted that the variability in activity was not significantly reduced between different HLM donors, as might have been expected if each donor sample contained an individual and varied concentration of fatty acids (Fig. 4). We hypothesized that this is due to the lack of donor-dependent fatty acid concentrations present in the individual donor microsomal incubations, although the concentrations and identified fatty acids released during incubation were not investigated. Therefore, we considered that the inclusion of BSA was not advantageous when considering the simultaneous characterization of microsomal glucuronidation activities of hepatic UGT isoforms. This is especially true for new drug molecules of moderate to high lipophilicity, as the greatly enhanced protein binding significantly outweighed the 2- to 3-fold activity enhancement of a small number of UGT enzyme activities and especially if intrinsic clearance of parent drug is the main measurement. Therefore, high total glucuronidation activity tools may be more suitable for the characterization of metabolically stable new drug candidates especially when the effect of BSA binding and the identity of the UGT enzymes involved in metabolism have not yet been determined, and where BSA-related binding may substantially diminish metabolism measurability.

\section{Conclusions}

To date, few in vitro UGT profiling studies have been reported using conditions optimized for such a wide variety of UGT isoform activities. In addition, only small numbers of positive control compounds are routinely deployed as markers of UGT activity in phenotyping experiments. In a pharmaceutical industry setting, use of the proposed substrates panel will allow control of multiple glucuronidation reactions in the automated screens used for compound optimization. The substrate cassetting approach enables more data to be generated using the same amount of (sometimes scarce) biologic material and analytical resources. Automated glucuronidation assays making use of these control cassettes and optimized experimental settings also enable more effective performance of multiple-donor activity studies, such as for correlation analyses, ontogeny investigations, or assessment of the effects of UGT polymorphism on drug clearance.

\section{Acknowledgments}

Protein binding experiments for trifluoperazine, propofol, amitriptyline, testosterone, and 5-hydroxytryptofol were conducted under contract by Synlab AG. We thank Dr. Massimiliano Donzelli and Sandrine Simon for organizing these studies. Protein binding experiments for the remaining substrates were conducted at Hoffmann-La Roche by Florian Klammers.

\section{Authorship Contributions}

Participated in research design: Badée, Qiu, Parrott, Collier, Schmidt, Fowler.

Conducted experiments: Badée, Qiu.

Performed data analysis: Badée, Qiu, Fowler.

Wrote or contributed to the writing of the manuscript: Badée, Qiu, Parrott, Collier, Schmidt, Fowler.

\section{References}

Badée J, Fowler S, de Wildt SN, Collier AC, Schmidt S, and Parrott N (2018) The ontogeny of UDP-glucuronosyltransferase enzymes, recommendations for future profiling studies and application through physiologically based pharmacokinetic modelling. Clin Pharmacokinet DOI: 10.1007/s40262-018-0681-2 [published ahead of print].

Court MH, Duan SX, Guillemette C, Journault K, Krishnaswamy S, Von Moltke LL, and Greenblatt DJ (2002) Stereoselective conjugation of oxazepam by human UDPglucuronosyltransferases (UGTs): S-oxazepam is glucuronidated by UGT2B15, while R-oxazepam is glucuronidated by UGT2B7 and UGT1A9. Drug Metab Dispos 30:1257-1265.

Di L and Obach RS (2015) Addressing the challenges of low clearance in drug research. AAPS J 17:352-357.

Dixit V, Hariparsad N, Desai P, and Unadkat JD (2007) In vitro LC-MS cocktail assays to simultaneously determine human cytochrome P450 activities. Biopharm Drug Dispos 28: $257-262$.

Donato MT, Montero S, Castell JV, Gómez-Lechón MJ, and Lahoz A (2010) Validated assay for studying activity profiles of human liver UGTs after drug exposure: inhibition and induction studies. Anal Bioanal Chem 396:2251-2263.

Fallon JK, Neubert H, Goosen TC, and Smith PC (2013) Targeted precise quantification of 12 human recombinant uridine-diphosphate glucuronosyl transferase $1 \mathrm{~A}$ and $2 \mathrm{~B}$ isoforms using nano-ultra-high-performance liquid chromatography/tandem mass spectrometry with selected reaction monitoring. Drug Metab Dispos 41:2076-2080.

Gagez A-L, Rouguieg-Malki K, Sauvage F-L, Marquet P, and Picard N (2012) Simultaneous evaluation of six human glucuronidation activities in liver microsomes using liquid chromatography-tandem mass spectrometry. Anal Biochem 427:52-59.

Gill KL, Houston JB, and Galetin A (2012) Characterization of in vitro glucuronidation clearance of a range of drugs in human kidney microsomes: comparison with liver and intestinal glucuronidation and impact of albumin. Drug Metab Dispos 40:825-835.

Gradinaru J, Romand S, Geiser L, Carrupt P-A, Spaggiari D, and Rudaz S (2015) Inhibition screening method of microsomal UGTs using the cocktail approach. Eur J Pharm Sci 71:35-45.

Hutzler JM and Tracy TS (2002) Atypical kinetic profiles in drug metabolism reactions. Drug Metab Dispos 30:355-362.

Joo J, Lee B, Lee T, and Liu K-H (2014) Screening of six UGT enzyme activities in human liver microsomes using liquid chromatography/triple quadrupole mass spectrometry. Rapid Commun Mass Spectrom 28:2405-2414.

Kariv I, Cao H, and Oldenburg KR (2001) Development of a high throughput equilibrium dialysis method. J Pharm Sci 90:580-587.

Kemp DC, Fan PW, and Stevens JC (2002) Characterization of raloxifene glucuronidation in vitro: contribution of intestinal metabolism to presystemic clearance. Drug Metab Dispos 30:694-700.

Lu D, Xie Q, and Wu B (2017) N-glucuronidation catalyzed by UGT1A4 and UGT2B10 in human liver microsomes: assay optimization and substrate identification. J Pharm Biomed Anal 145 692-703.

Mano Y, Usui T, and Kamimura H (2007) The UDP-glucuronosyltransferase 2B7 isozyme is responsible for gemfibrozil glucuronidation in the human liver. Drug Metab Dispos 35: 2040-2044.

Margaillan G, Rouleau M, Klein K, Fallon JK, Caron P, Villeneuve L, Smith PC, Zanger UM, and Guillemette C (2015) Multiplexed targeted quantitative proteomics predicts hepatic glucuronidation potential. Drug Metab Dispos 43:1331-1335.

Miyagi SJ and Collier AC (2011) The development of UDP-glucuronosyltransferases 1A1 and 1A6 in the pediatric liver. Drug Metab Dispos 39:912-919.

Patten CJ (2006) New technologies for assessing UDP-glucuronosyltransferase (UGT) metabolism in drug discovery and development. Drug Discov Today Technol 3:73-78.

Pillai VC, Strom SC, Caritis SN, and Venkataramanan R (2013) A sensitive and specific CYP cocktail assay for the simultaneous assessment of human cytochrome $\mathrm{P} 450$ activities in primary cultures of human hepatocytes using LC-MS/MS. J Pharm Biomed Anal 74:126-132.

Rowland A, Gaganis P, Elliot DJ, Mackenzie PI, Knights KM, and Miners JO (2007) Binding of inhibitory fatty acids is responsible for the enhancement of UDP-glucuronosyltransferase 2B7 activity by albumin: implications for in vitro-in vivo extrapolation. J Pharmacol Exp Ther 321 137-147.

Rowland A, Knights KM, Mackenzie PI, and Miners JO (2008) The "albumin effect" and drug glucuronidation: bovine serum albumin and fatty acid-free human serum albumin enhance the glucuronidation of UDP-glucuronosyltransferase (UGT) 1A9 substrates but not UGT1A1 and UGT1A6 activities. Drug Metab Dispos 36:1056-1062.

Rowland A, Miners JO, and Mackenzie PI (2013) The UDP-glucuronosyltransferases: their role in drug metabolism and detoxification. Int J Biochem Cell Biol 45:1121-1132.

Seo K-A, Kim H-J, Jeong ES, Abdalla N, Choi C-S, Kim D-H, and Shin J-G (2014) In vitro assay of six UDP-glucuronosyltransferase isoforms in human liver microsomes, using cocktails of probe substrates and liquid chromatography-tandem mass spectrometry. Drug Metab Dispos 42 : $1803-1810$.

Slovak JE, Mealey K, and Court MH (2017) Comparative metabolism of mycophenolic acid by glucuronic acid and glucose conjugation in human, dog, and cat liver microsomes. $J$ Vet Pharmacol Ther 40:123-129.

Spaggiari D, Geiser L, Daali Y, and Rudaz S (2014) A cocktail approach for assessing the in vitro activity of human cytochrome P450s: an overview of current methodologies. J Pharm Biomed Anal 101:221-237. 
Thakare R, Alamoudi JA, Gautam N, Rodrigues AD, and Alnouti Y (2018) Species differences in bile acids II. Bile acid metabolism. $J$ Appl Toxicol 38:1336-1352.

Tukey RH and Strassburg CP (2000) Human UDP-glucuronosyltransferases: metabolism, expression, and disease. Annu Rev Pharmacol Toxicol 40:581-616.

Vanstraelen K, Wauters J, De Loor H, Vercammen I, Annaert P, Lagrou K, and Spriet I (2014) Protein-binding characteristics of voriconazole determined by high-throughput equilibrium dialysis. J Pharm Sci 103:2565-2570.

Walia G, Smith AD, Riches Z, Collier AC, and Coughtrie MWH (2018) The effects of UDPsugars, UDP and $\mathrm{Mg}^{2+}$ on uridine diphosphate glucuronosyltransferase activity in human liver microsomes. Xenobiotica 48:882-890.

Walsky RL, Bauman JN, Bourcier K, Giddens G, Lapham K, Negahban A, Ryder TF, Obach RS, Hyland R, and Goosen TC (2012) Optimized assays for human UDP-glucuronosyltransferase (UGT) activities: altered alamethicin concentration and utility to screen for UGT inhibitors. Drug Metab Dispos 40:1051-1065.
Zientek MA and Youdim K (2015) Reaction phenotyping: advances in the experimental strategies used to characterize the contribution of drug-metabolizing enzymes. Drug Metab Dispos 43 $163-181$.

Address correspondence to: Stephen Fowler, Pharmaceutical Sciences, Roche Pharma Research and Early Development, Roche Innovation Centre Basel, Grenzacherstrasse 124, 4070 Basel, Switzerland. E-mail: stephen.fowler@roche. com; or Justine Badée, Department of Pharmaceutics, Center for Pharmacometrics and Systems Pharmacology, University of Florida at Lake Nona, Orlando, FL 32827. E-mail: jbadee@cop.ufl.edu 